\title{
Reliability and Validity of Compulsive Buying Scale Without Middle Point
}

\author{
SantanuChoudhury $^{\mathrm{a}}$, DibyojyotiBhattacharjee ${ }^{\mathrm{b}}$ \\ $\mathrm{a}^{*}$ Department of Statistics, Gurucharan College, Silchar, Assam,India \\ ${ }^{\mathrm{b}}$ Department of Statistics, Assam University, Silchar, Assam, India \\ a*Corresponding Author Email: choudhuryshantanu11@gmail.com
}

Article History: Received: 10 November 2020; Revised 12 January 2021 Accepted: 27 January 2021; Published online: 5 April 2021

\begin{abstract}
Objective: The study aims at finding out the affect of reliability and validity for the compulsive buying behavior scale by Valence, d' Astou's and Fortier Scale without middle point.

Methodology: Responses across 5 to 9 point scales are obtained to calculate the reliability and validity of compulsive buying behavior scale by Valence, d' Astou's and Fortier Scale. Cronbach's alpha is used to measure the internal reliability of the scale. To compare the reliability coefficients among different scale points Feldt test and Hakstian-Whalen test are used. Convergent validity for measuring inter-correlations between scales with different numbers of response categories is used and Fisher's $-\mathrm{r}$ to $-\mathrm{z}$ transformation is used to test population correlation coefficient.

Conclusion: From study it is concluded that there is no change in reliability and validity when the middle point is dropped from the compulsive buying scale.

Keywords: Valence, d' Astou's and Fortier Scale, Cronbach's Alpha, Feldt Test, Hakstian-Whalen Test, Correlation Coefficient, Convergent Validity
\end{abstract}

\section{Introduction}

The main purpose of a rating scale is to allow respondents to express both the direction and strength of their opinion about a topic. The widespread use of rating scales in market and social research has generated considerable debate over the optimal number of scale points to use.

Typically, market researchers would prefer respondents to make a definite choice rather than choose neutral or intermediate positions on a scale. For this reason, a scale without a midpoint would be preferable, provided it does not affect the validity or reliability of the responses.

While many authors have concluded that the optimal number of scale categories is content specific and a function of the conditions of measurement (Komorita 1963; Matell\& Jacoby 1971; Wildt\&Mazis 1978; Cox 1980; Friedman, Wilamowsky, \& Friedman 1981), few published studies have addressed the issue of whether or not a mid-point category should be included on rating scales viz. compulsive buying scale by Valence, d' Astou's and Fortier Scale (1988).

Matell and Jacoby (1972) demonstrated that as the number of scale steps is increased, respondents' use of the mid-point category decreases. For instance, their three and five point scale formats were associated with an average of $20 \%$ of respondents choosing the mid-point category whereas this category was utilized by only an average of $7 \%$ of respondents scoring seven, nine..., nineteen category formats. The authors, Matell and Jacoby's (1972) advice on minimizing usage of the mid-point category was to either not include it at all or use scales with many points. They concluded by stating that "the decision would seem to depend on the level of 'uncertain 'responses one is willing to tolerate".

Worcester and Burns (1975) included a balanced four point Likert scale without mid-point in their major examination of the precision of verbal tags. Their study highlighted the implications of scoring verbal scales by the traditional practice of +2 to -2 or 5, 4, 3,2, 1 in the light of their finding that grammatically balanced Likert scales are often unbalanced in interpretation; for instance, 'tend to disagree' is not directly opposite 'tend to agree'. Worcester and Burns also concluded that a four point scale without a mid-point appears to push more respondents towards the positive end of the scale.

However, some researchers claim that the use of mid-points on Likert type scale viz., compulsive buying scale may affect research reliability and validity, but some other researchers disagree. 
Nevertheless, some researchers argue that the use of reliability as a criterion to judge the merit of midpoints is inappropriate (Chang, 1994). As Cronbach (1950) already notes, "there is no merit in enhancing test reliability unless validity is enhanced at least proportionately." In other words, validity should be a better criterion than reliability (Chang, 1994). Some studies evaluate the impacts of midpoints on measurement validity. However, the findings are also contradictory. For instance, some studies find that the construct validity may not be influenced by the midpoints (Adelson\&McCoach, 2010; Kulas, Stachowski, \& Haynes, 2008), but some researchers suggest the omission of the midpoints may impair the validity (Johns, 2005) ( https://www. rangevoting.org/ MB_ V2_ N3_ Garland.pdf).

The researchers are interested to study the compulsive behavior of consumers. Thus, it is important to decide about the affect of mid-point on reliability and validity for compulsive buying behavior scale. To deal with these affect we shall use the compulsive buying behavior scale by Valence, d' Astou's and Fortier Scale (1988).

\section{Objective}

In the review of literature, it is identified the importance of studying the compulsive behavior of the consumers in marketing. It is also very pertinent how the middle point affect the reliability and validity of scales. So there is a gap in the literature regarding the optimal number of scale points in the said compulsive scale. Thus, the paper is designed to find the most compatible scale point for the compulsive buying behavior scale by Valence, d'Astou's and Fortier Scale without middle point.

\section{Hypotheses}

To attain the objective the hypotheses are as follows:

(i) $\mathrm{H}_{01}$ : There is no significant difference with regard to correlation coefficient between different scale points without middle point in the Valence, d' Astou's and Fortier compulsive scale

(ii) $\mathrm{H}_{02}$ : There is no significant difference with regard to Cronbach- $\alpha$ value between different scale points without middle point in the Valence, d' Astou's and Fortier compulsive scale

\section{Methodology}

The methodology which is adopted to find the affect when the middle point is dropped from the scales is as follows. For the purpose of assessing reliability we shall use Cronbach alpha reliability and to test the significance difference among various alpha coefficients we shall use the Feldt's test (1980) and Hakstian-Whalen test (1976).

The population selected is an urban population belonging to Silchar town of Assam, India. Considering the population size of Silchar (1.72 lakh as per 2011 census) a sample of size 196 consumers is randomly selected for the study. The sample of the said size can provide results with 95 percent confidence level and a standard error of 7 percent.

There are 13 statements in the Compulsive Buying Scale given by Valence, d' Astou's and Fortier Scale (1988). The researcher obtained responses on various scale points used i.e., 5-point scale to 9-point scale from a sample of 196 respondents using Compulsive Buying Scale (Given in Appendix).In this case all the point scale were supported by the verbal descriptors in a way that there were no middle point in the scale.The sample of size 196 was drawn by systematic random sampling and the place of study was Big Bazar, Silchar Assam, India. The respondents took four to five weeks' time to complete the 5 set of questionnaires e.g. the respondent took one week time to collect the information with the first set of questionnaire i.e. the questionnaire with the 5-point scale. The second questionnaire e.g. the questionnaire with 6-point scale was issued after the receipt of first questionnaire in the second week. Similarly, the other sets of questionnaires were issued.

\subsection{Cronbach Alpha as a Measure of Internal Reliability of a Scale}

Cronbach's alpha reliability (Cronbach, 1951) is one of the most widely used measures of reliability in the social and organizational sciences. Cronbach's alpha reliability describes the reliability of a sum (or average) of q measurements where the $q$ measurements may represent qraters, occasions, alternative forms, or questionnaire/test items. When the measurements represent multiple questionnaire/test items, which is the most common application, Cronbach's alpha is referred to as a measure of "internal consistencyreliability. If the measurements are "parallel(McDonald, 1999), they will have equal variances and equal covariances. As an example, suppose that we have a five-item questionnaire $(q=5)$ to measure employee work ethic with each item scored on a 1-7 Likert scale. In this example, the parallel measurement assumption requires all five items to have equal variances and all 5(4)/2 = 10 covariances among the five items are required to be equal. A tau-equivalent assumption does not require the five items to have equal variances but does require the 10 covariances to be equal. With a congeneric assumption, the variances of the five items can be unequal and the 10 covariances can be unequal. 
Sijtsma (2009) has criticized the widespread use of Cronbach's alpha because it understates the reliability of the sum or average of the $q$ measurements if the essentially tau-equivalent assumption is not satisfied. However, this criticism may be too harsh because the degree of understatement will be small in typical applications where the measurements are well approximated by a one-factor model and the factor loadings for the measurements are not highly dissimilar. Many $q$-item congeneric measurements have standardized factor loadings that are not too dissimilar (e.g., ranging from about .5 to .8) because items with small loadings or items that load on a second factor are typically discarded during scale development and Cronbach's alpha will only slightly understate the reliability of the scale in these situations. Furthermore, the tau-equivalent assumption is realistic in applications where the $q$ measurements represent alternative forms of a placement test or the ratings of properly trained raters. The essentially tau-equivalent assumption will be violated in applications where the measurements are not all measured using the same metric, for example, if some items are scored agree/disagree while other items are scored 1 to 7 . In these applications, it may be possible to transform congeneric measures into approximate essentially tauequivalent measurements by simply rescaling some of the measurements. The sample value of Cronbach's alpha contains sampling error of unknown direction and unknown magnitude. An acceptable reliability value depends on the type of application, and furthermore, the focus should be on the population reliability value and not on the sample reliability value.

If there is only one test administration researchers may resort to, in the context of classical test theory (Lord and Novick 1968), internal consistency coefficients for estimating the reliability of the test. The most commonly used consistency coefficients are Cronbach's alpha and the Spearman-Brown formula (Cortina 1993; Osburn 2000; Hogan et al. 2000; Feldt and Charter 2003; Grayson 2004; Warrens 2014, 2015). Internal consistency coefficients estimate reliability by dividing the total test into parts. A test may already consist of multiple parts, for example, a multiple choice part and an essay part. If the test consists of a set of items, the parts can be the individual items or subsets of the items. All reliability coefficients are based on the assumption that the different parts are homogeneous in content (Feldt and Brennan 1989) (https://www.researchgate.net/ publication 1266798982).

Analysis for reliability coefficient is given in Table 1.

\subsection{Test for Comparing Reliability Coefficients}

In measurement studies the researcher may wish to test the hypothesis that Cronbach's alpha reliability coefficient is the same for two measurement procedures. If there is only one test administration researchers may resort to, in the context of classical test theory (Lord and Novick 1968), internal consistency coefficients for estimating the reliability of the test. The most commonly used consistency coefficients are Cronbach's alpha and the Spearman-Brown formula (Cortina 1993; Osburn 2000; Hogan et al. 2000; Feldt and Charter 2003; Grayson 2004; Warrens2014, 2015).Internal consistency coefficients estimate reliability by dividing the total test into parts(https://link.springer.com/article /10.1007/s11634-015-0198-6).

A statistical test exists for independent samples of subjects is Feldt (1980) test which is

$$
W=\frac{1-\hat{\alpha}_{2}}{1-\hat{\alpha}_{1}} \text {. }
$$

and we evaluate $W$ as an $F$ statistic with $\left(n_{1}-1\right),\left(n_{2}-1\right)$ df.

In general, a test is said to be reliable if it produces similar scores for participants under consistent conditions. In reliability estimation a researcher wants to reflect the impact of as many sources of measurement error as possible (Feldt and Brennan 1989).

To test that $k$ coefficients are identical, we use the Hakstian-Whalen test (1976) (https://www.google.com/search?ei =_HRCYM7EA9uQ4-EPnOWziAQ\&q):

$$
M=\sum_{j=1}^{k} A_{j}-\frac{\sum_{j=1}^{k} A_{j}\left(1-\hat{\alpha}_{j}\right)^{-1 / 3}}{\sum_{j=1}^{k} A_{j}\left(1-\hat{\alpha}_{j}\right)^{-2 / 3}}, \text { where } A_{j}=\frac{\left(n_{j}-1\right)(9 N-11)^{2}}{18 n_{j}\left(N_{j}-1\right)}
$$

Here we note that $n_{j}$ is the number of items on the $j^{\text {th }}$ test and $N_{j}$ is the number of persons taking the $j^{\text {th }}$ test and then we evaluate $M$ as a $\chi^{2}$ on $K$ - $l$ d.f.Feldt's test result for different scale points is shown in the Table 2. 


\subsection{Validity Test: Convergent validity for measuring inter-correlations between scales with different numbers of response categories}

Convergent validity is one of the topics related to construct validity (https:// doi.org /10.1007 /978-94-0070753-5_539) (Gregory, 2007). Convergent validity can be estimated using correlation coefficients. Convergent validity states that tests having the same or similar constructs should be highly correlated. Two methods are often applied to test convergent validity. One is to correlate the scores between two assessment tools or tools' sub-domains that are considered to measure the same construct.

Convergent and discriminant validities are two fundamental aspects of construct validity. Convergent validity refers to how closely the new scale is related to other variables and other measures of the same construct. Not only should the construct correlate with related variables but it should not correlate with dissimilar, unrelated ones. A determination along the latter lines is referred to as discriminant validity (de Vet et al., 2011; Streiner et al., 2015) (https://www .sciencedirect.com/science/ article /pii/ B97801 2801504 9000076). Convergent validity for measuring inter-correlations between scales with different numbers of response categories are shown in the Table1.Using Fisher's-r to $-\mathrm{z}$ transformation (https://blogs.gwu.edu/weissba/teaching /calculators/fishers-ztransformation/) the significant difference between scale points is found out.

\subsection{Fisher's $-r$ to $-z$ transformation}

Fisher $z$-transformation can be used to test hypotheses about the value of the population correlation coefficient $\rho$ between variables $X$ and $Y$. This is because, when the transformation is applied to the sample correlation coefficient, the sampling distribution of the resulting variable is approximately normal, with a variance that is stable over different values of the underlying true correlation(https://en. wikipedia.org /wiki/Fisher transformation).

Let us suppose that given a set $n$ bi-variate sample pairs $\left(X_{i}, Y_{i}\right), i=1,2, \ldots \ldots, n$, the sample correlation coefficient $r$ is given by

$$
r=\frac{\operatorname{Cov}(X, Y)}{\sigma_{X} \sigma_{Y}}=\frac{\sum_{i=1}^{n}\left(X_{i}-\bar{X}\right)\left(Y_{i}-\bar{Y}\right)}{\sqrt{\sum_{i=1}^{n}\left(X_{i}-\bar{X}\right)^{2}} \sqrt{\sum_{i=1}^{n}\left(Y_{i}-\bar{Y}\right)^{2}}}
$$

where $\operatorname{Cov}(X, Y)$ denotes the covariance between the variables $X$ and $Y$ and $\sigma$ stands for the standard deviation of the respective variable.

Fisher's z- transformation of $r$ is defined as

$$
z=\frac{1}{2} \log \left(\frac{1+r}{1-r}\right)=\arctan h(r)
$$

wherearctanhis the inverse hyperbolic tangent function.

If $(X, Y)$ has a bi-variate normal distribution with correlation coefficient $\rho$ and the pairs $\left(X_{i}, Y_{i}\right)$ are independently and identically distributed, then the variate $z$ is approximately normally distributed with mean

$$
\frac{1}{2} \log \left(\frac{1+r}{1-r}\right)
$$

and standard error

$$
\frac{1}{\sqrt{n-3}}
$$

Where $n$ is the sample size and $\rho$ is the true correlation coefficient.

The behavior of this transform has been extensively studied since Fisher introduced it in 1915. Fisher himself found the exact distribution of $z$ for data from a bivariate normal distribution in 1921; Gayen in1951 determined the exact distribution of $z$ for data from a bivariate Type AEdgeworth distribution. Hotelling in 1953 calculated the Taylor series expressions for the moments of $z$ and several related statistics and Hawkins in 1989 discovered 
the asymptotic distribution of $z$ for data from a distribution with bounded fourth moments (https://en.wikipedia.org/wiki/Fisher_transformation).

\section{Result and Findings}

Based on the methodology discussed in Section 4, the computations are done and are placed in the tables below.

Table 1.Analysis for Reliability Coefficient

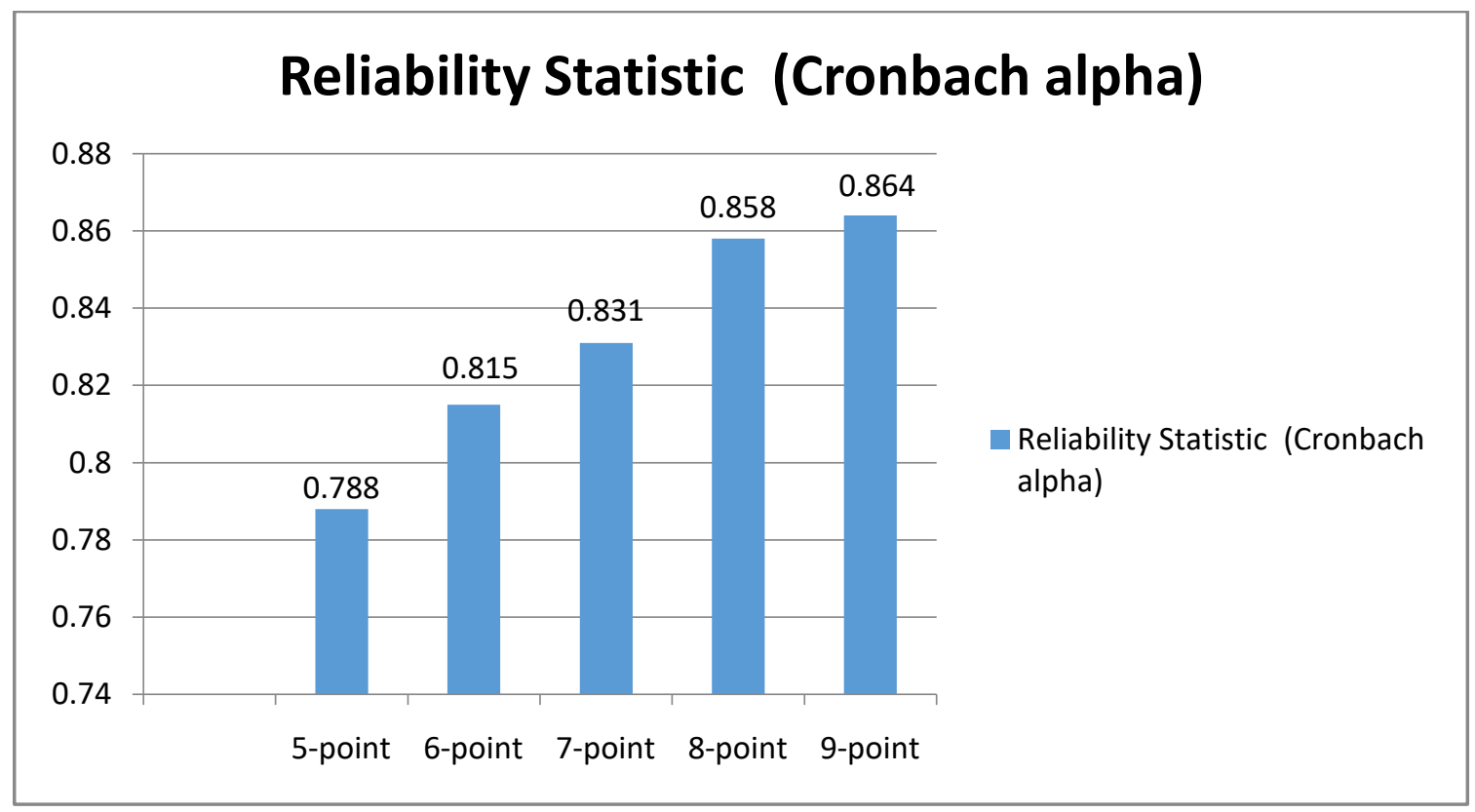

From Table1, which indicates that the reliability coefficients are all relatively high ( 0.78 or above), so it is found that as the number of scale point increases the alpha coefficient also increases.

Table 2. Feldt's Test Result

\begin{tabular}{|l|l|l|l|l|}
\hline $\begin{array}{l}\text { Reliability of scale } \\
\text { points compared }\end{array}$ & Reliability Values & W statistic & p-value & Comment \\
\hline 5,6 & $0.788,0.815$ & 1.1277 & 0.7244 & Not significant \\
\hline 6,7 & $0.815,0.831$ & 0.9135 & 0.3268 & Not significant \\
\hline 7,8 & $0.831,0.858$ & 1.9135 & 0.8060 & Not significant \\
\hline 8,9 & $0.858,0.864$ & 0.9577 & 0.4152 & Not significant \\
\hline
\end{tabular}

From Table 2, it can thus be stated that none of the differences between the alpha coefficients for $(5,6),(6,7)$, $(7,8)$ and $(8,9)$ - point are statistically significant: using $p>.05$, so there is no effect of mid-point on reliability of scales.

Table3. Convergent Validity: Inter-correlations between Scales with Different Numbers of Response Categories

\begin{tabular}{|l|l|l|l|l|l|}
\hline Categories & $\mathbf{5}$ & $\mathbf{6}$ & $\mathbf{7}$ & $\mathbf{8}$ & $\mathbf{9}$ \\
\hline $\mathbf{5}$ & & & & & \\
\hline $\mathbf{6}$ & 0.909752 & & & & \\
\hline $\mathbf{7}$ & 0.898404 & 0.908114 & & & \\
\hline $\mathbf{8}$ & 0.831089 & 0.876416 & 0.913779 & & 0.903468 \\
\hline $\mathbf{9}$ & 0.846932 & 0.852469 & 0.877063 & & \\
\hline
\end{tabular}

From Table 3, it is seen that every scale is correlated highly with each of the others, and all of the correlation coefficients. These results, which provide evidence of convergent validity, also indicate that the scales with relatively more response categories correlated best with one another. This table also indicates that the correlation coefficient between the scale points goes on decreasing as distance between the scale point increases. 
Let $\rho_{k}$ and $\rho_{k}$,denote the correlation between different pair of scale points when the $k$-point Valence, d' Astou's and Fortier compulsive scale is used. Here, we would be interested to test if the inter-correlation between different pair of scale points by the Valence, d' Astou's and Fortier compulsive scale differ significantly or not. Thus, the null hypothesis to test is,

$$
\mathrm{H}_{01}: \rho_{k}=\rho_{k^{\prime}}
$$

The test is performed against the alternative hypothesis that

$\mathrm{H}_{11}$ : Not all inter-correlation pairs are equal.

To test $\mathrm{H}_{01}$, we shall apply Fisher's $-r$-to $-z$ transformation as described earlier.

Table 4.Validity Test Result: Fisher's $-\mathrm{r}$-to $-\mathrm{z}$ transformation:

\begin{tabular}{|l|l|l|l|l|}
\hline $\begin{array}{l}\text { Inter-correlation of } \\
\text { scale } \\
\text { compared }\end{array}$ & Coints & z-value & p-value & Comment \\
\hline$(5,6),(6,7)$ & $(0.9098,0.9081)$ & 0.07 & 0.9442 & Not significant \\
\hline$(6,7),(7,8)$ & $(0.9081,0.9138)$ & 0.23 & 0.8181 & Not significant \\
\hline$(7,8),(8,9)$ & $(0.9138,0.9035)$ & 0.41 & 0.6818 & Not significant \\
\hline
\end{tabular}

From Table 4 ,it is observed that the correlation coefficient between $(5,6)$ point is 0.9098 and that of $(6,7)$ point is 0.9081 , using the Fisher $r$-to- $z$ transformation to assess the significance of the difference between two correlation coefficients, we calculate a value of $\mathrm{z}$ which is $0.07(p$-value is $0.9442>0.05$ ) and is not significant at $5 \%$ level. The correlation coefficient between $(6,7)$ point is 0.9081 and that of $(7,8)$ point is 0.9138 , using the Fisher $r$-to- $z$ transformation to assess the significance of the difference between two correlation coefficients, we calculate a value of $\mathrm{z}$ which is 0.23 ( $p$--value is $0.8181>0.05$ ) and is not significant at $5 \%$ level. The correlation coefficient between $(7,8)$ point is 0.9138 and that of $(8,9)$ point is 0.9035 , using the Fisher $r$-to- $z$ transformation to assess the significance of the difference between two correlation coefficients, we calculate a value of $\mathrm{z}$ which is 0.41 ( $p$-value is $0.6818>0.05$ ) and is not significant at $5 \%$ level. Thus it can be concluded that there is no effect of midpoint on the validity of scale.

\section{Conclusion}

This study focuses on the affect of Reliability and Validity of Compulsive Buying Scale. This study shows that

(i) The reliability coefficients are all relatively high ( 0.78 or above), it is found that as the number of scale point increases the alpha coefficient is also increases.

(ii) From Feldt's test, it is observed that none of the differences between the alpha coefficients for $(5,6)$, $(6,7),(7,8)$ and $(8,9)$ - point are statistically significant: using $p>.05$.

(iii) Every scale correlated highly with each of the others, and all of the correlation coefficients. These results, which provide evidence of convergent validity, also indicate that the scales with relatively more response categories correlated best with one another. It is also observed that the correlation coefficient between the scale points goes on decreasing as distance between the scale point increases.

(iv) The correlation coefficient between $(5,6)$ point is 0.9098 and that of $(6,7)$ point is 0.9081 , using the Fisher $r$-to- $z$ transformation to assess the significance of the difference between two correlation coefficients, we calculate a value of $\mathrm{z}$ which is 0.07 ( $p$-value is $0.9442>0.05)$ and is not significant at $5 \%$ level. The correlation coefficient between $(6,7)$ point is 0.9081 and that of $(7,8)$ point is 0.9138 , using the Fisher $r$-to- $z$ transformation to assess the significance of the difference between two correlation coefficients, we calculate a value of $\mathrm{z}$ which is 0.23 ( $p$-value is $0.8181>0.05$ ) and is not significant at $5 \%$ level. The correlation coefficient between $(7,8)$ point is 0.9138 and that of $(8,9)$ point is 0.9035 , using the Fisher r-to-z transformation to assess the significance of the difference between two correlation coefficients, we calculate a value of $\mathrm{z}$ which is 0.41 ( $p$-value is $0.6818>0.05$ ) and is not significant at $5 \%$ level .Thus it can be concluded that there is no effect of mid-point on the validity of scale.

Thus it can be concluded that there is no change in reliability and validity when the middle point is dropped from the compulsive buying scale

\section{References}

Bonett, D. G., \& Wright, T. A. (2014). Cronbach's alpha reliability: Interval estimation, hypothesis testing, and sample size planning. Journal of Organizational Behavior (https:// www. researchgate.net / publication/ 266798982). 
Chin CL., Yao G. (2014) Convergent validity. in: Michalos A.C. (eds.) encyclopedia of quality of life and wellbeing research. Springer, Dordrecht (https:// doi.org / 10.1007/ 978-94-007-0753-5_573).

Cronbach, L. J. (1951). Coefficient alpha and the interval structure of tests. Psychometrika, 16, 297334(https://link.springer.com/article/10.1007\% 2FBF02310555).

Feldt, L. S. (1980). A Test of the hypothesis that Cronbach's alpha reliability coefficient is the same for Two Tests Administered to the Same Sample.Psychometrika, 45, 99-105 (https://psycnet. apa.org/record/198109335-001).

Fisher, R. A. (1915). Frequency distribution of the values of the correlation coefficient in samples of an indefinitely large population.Biometrika. 10 (4): 507-521 doi: 10.2307 /2331838 hdl: 2440/15166. JSTOR

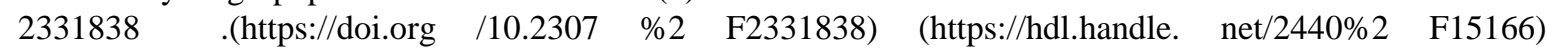
(https://www.jstor .org/stable /2331838).

Fisher, R. A. (1921). On the 'probable error' of a coefficient of correlation deduced from a small sample (http://digital.library.adelaide.edu.au/ dspace /bitstream /2440/15169/1/14.pdf) (PDF). Metron.1: 3-32.

Garland, R. (1991). The mid-point on a rating scale: Is it desirable? Marketing Bulletin, 2, 66-70.( https://www. rangevoting.org/ MB_V2_N3_Garland.pdf)

Gayen, A. K. (1951). The frequency distribution of the product-moment correlation coefficient in random samples of any size drawn from non-normal universes.Biometrika. 38 (1/2): $219-247$. doi:10.1093/biomet/38.1-2.219. JSTOR 2332329.(https://doi.org/10.1093\%2Fbiomet\%2F38.1-2.219)

Gregory, R. J. (2007). Psychology testing: History, principles and applications (5th ed.). Boston: Ally \& Bacon. (https:// doi.org /10.1007 /978-94-007-0753-5_539)

Hakstian, A. R., \& Whalen, T. E.(1976). A k-sample significance test for independent alpha coefficients.Psychometrika, 41(2), 219-231. ( https:// doi.org/10.1007/ BF0 2291840

Hotelling, H. (1953). New light on the correlation coefficient and its transforms. Journal of The Royal Statistical Society, Series B. 15 (2): 193-225. JSTOR 2983768 (https://www.jstor.org/stable/ 298376 2/15/2021 Fisher transformation - Wikipedia https://en.wikipedia.org/wiki /Fisher_transformation 4/4 8)

Matell, M.S. \& Jacoby, J. (1972).Is there an optimal number of alternatives for Likert scale items? Effects of testing time and scale properties. Journal of Applied Psychology, 56 (6), 506-509.

McDonald, R. P. (1999). Test theory: A unified treatment. Mahwah, NJ: LEA ( https://scholar.google.co.in/scholar?q)

Valence G, d'Astous A, Fortier L. (1988). Compulsive Buying: Concept and Measurement. J. Consumer Policy. 11: 419-433 African Journal of Business Management Vol. 5(14), pp. 5424-5434.

Weiss, B.A. (2011). Fisher's r-to-z transformation calculator to compare two independent Samples [Computer Software]. (https://blogs. gwu.edu /weissba /teaching/calculators/ shers-z-transformation/ ) .

\section{Appendix :Compulsive Buying Scale}

\section{(Valence, d' Astou's and Fortier Scale)}

1. When I have money, I cannot help but spend part or the whole of it.

2. I am often impulsive in my behavior.

3. For me, shopping is a way of facing the stress of my daily life and of relaxing.

4. I sometimes feel that something inside pushed me to go shopping.

5. There are times when I have a strong urge to buy (clothing, books, etc.)

6. At times I have felt somewhat guilty after buying a product, because it seemed unreasonable.

7. There are some things I buy that I do not show to anybody for fear of being perceived as irrational in my buying behavior ("a foolish expense").

8. I often have an unexplainable urge, a sudden and spontaneous desire, to go and buy something in the store.

9. As soon as I enter a shopping centre I have an irresistible urge to go in to shop and buy something.

10. I am one of those people who often respond to direct mail offers (e.g., books, records).

11. I have often bought a product that I did not need, while knowing that I have very little money left.

12. I am a spend thrift.

13. I have sometimes thought, "If I had to do it all over again, I would ......." and felt sorry for something I have done or said. 